\title{
Comparative study of management of olecranon fractures using tension band wiring technique with cancellous screw and $K$ wires
}

\author{
Krishnaprasad H. S. ${ }^{1}$, Shivanna ${ }^{2} *$ \\ ${ }^{1}$ Consultant Orthopaedic Surgeon, Ortho Clinic and Multispecialty Centre, Chamarajanagara, Karnataka, India \\ ${ }^{2}$ Senior resident, Department of Orthopaedics, CIMS, Chamarajanagara, Karnataka, India
}

Received: 25 July 2016

Accepted: 30 July 2016

\section{*Correspondence:}

Dr. Shivanna,

E-mail: dr.shivanna@gmail.com

Copyright: (c) the author(s), publisher and licensee Medip Academy. This is an open-access article distributed under the terms of the Creative Commons Attribution Non-Commercial License, which permits unrestricted non-commercial use, distribution, and reproduction in any medium, provided the original work is properly cited.

\begin{abstract}
Background: Olecranon fractures are commonly seen injuries in the emergency room. The surgical management of displaced fractures is open reduction and internal fixation with $\mathrm{k}$-wires and figure of eight tension band wiring. It can also be fixed with intramedullary cancellous screw with tension band wiring. The present study is to compare the results of both the surgical procedures and to assess the merits and demerits.

Methods: This prospective comparative study was carried out from August 2012 to November 2013 in Bapuji Hospital and Chigateri General Hospital attached to J.J.M Medical College, Davangere, India, where among 20 olecranon fractures, ten were treated with Tension band wiring with Kirshner wire and another ten treated with intramedullary cacellous screw with tension band wiring and the results were evaluated and compared.

Results: In our study, most of the cases were type II B fractures according to Colton's classification. According to Mayo elbow performance score, Excellent results found in 8 patients (80\%), good in1patient (10\%) and fair in 1 patient (10\%) in cancellous screw group and in $\mathrm{K}$ wire group, 5 patient (50\%) had excellent, 3 patient (30\%) had good and 2 patients $(20 \%)$ had fair results. No poor result was seen in both groups.

Conclusions: From this study it is concluded that using cancellous screw with tension band wiring for displaced transverse and oblique olecranon fractures gives better clinical results when compared to tension band wiring with Kwire fixation avoiding cost, work time loss and possible complications from hard ware removal.
\end{abstract}

Keywords: Olecranon fractures, Cancellous screw, Tension band wiring

\section{INTRODUCTION}

Olecranon fractures are one of the most commonly seen orthopaedic injuries in the emergency room. Fractures of the Olecranon process of the Ulna typically occurs as a result of a motor-vehicle or motorcycle accident, a fall, or assault. Undisplaced fractures can be treated with a short period of immobilization followed by gradually increasing range of motion. Many authors believe that undisplaced fractures where the displacement is less than $2 \mathrm{~mm}$ are best treated conservatively. ${ }^{1}$
When displaced, open reduction and internal fixation are usually required to obtain anatomical realignment of the articular surface and restore normal elbow function. The fixation should be stable, allow active elbow flexion and extension and promote union of the fracture. ${ }^{2}$

In the past, closed reduction and plaster cast application was the treatment for fracture of olecranon. But, prolonged immobilization with its own complications increased the morbidity and mortality of patients. ${ }^{3}$ Sachs reported excellent results with rapid resolution of 
function by dispensing with any form of splinting, allowing the arm to hang in extension, and instituting early massage. ${ }^{4}$ However, Elite reported a rapid return to relatively normal flexion and extension of the elbow after this treatment regimen, regardless whether fibrous or bony union. ${ }^{4}$ Daland presented the first substantial series of 48 cases of fracture olecranon treated conservatively. He concluded that close reduction was very unsatisfactory and that open reduction is invariably indicated. ${ }^{5}$ So keeping this in consideration, it has become important to intervene surgically. The active mobilization after surgery will restore the patient to normal function as early as possible. The early and active movement not only prevents the tissue from fracture disease but greatly influences the quality and rapidity of fracture union.

Stable internal fixation with figure-of-eight tension-band wire fixation for simple transverse fractures allows early motion to minimize stiffness. ${ }^{6}$ Hotchkirs, Robert N, preferred pure transverse fractures without comminution are ideally suited to tension band wiring. Weber and Vasey suggested that both limbs of figure of eight wire should be twisted in order to increase the rigidity of fixation. ${ }^{7}$ Cooper Jerald L, Robert D. and D'Ambrosia, according to them type II (transverse or oblique) fractures of olecranon, internal fixation options include tension band wiring with associated intramedullary wires or screw, longitudinal intramedullary fixation alone, and intra-fragmentary compression screws with or without neutralizing plate. They prefer parallel intramedullary Kirschner wire or a single $6.5 \mathrm{~mm}$ cancellous screw alone with figure of eight tension band wiring and they concluded that this method transforms tension forces into compressing forces across the fracture site. They also preferred intramedullary compression screw to prevent anterior subluxation in case of fracture dislocation injuries of elbow. ${ }^{8}$

The K-wire used in AO tension-band technique resists shearing force better than the figure of eight wire alone. So this gives a good result by converting tensile force to compressive at the fracture site., ${ }^{2,9,10}$

Cancellous screws plus wire combination is said to have provided the greater strength of fixation i.e., by converting tensile force to compressive force at fracture site with addition resistance to displacement by lag screw compression. ${ }^{11}$

Murphy D. F. et al, conducted an experimental studybiomechanical analysis of fixation methods; on fresh cadavar specimen model in the authors laboratory which was designed to mirror the clinical situation as closely as possible. In their study, the screw plus wire in combination provided the greatest strength of fixation to tensile forces applied to the fracture site. On a biomechanical basis, this method combines the tension band effect of converting tensile forces to compression forces at the fracture site with the additional resistance to displacement by lag screw compression. ${ }^{12}$ Fan, $\mathrm{Wu}$ and
Shin, did a study on olecranon fractures treated with tension band wiring techniques comparisons among three different configurations. They conducted retrospective comparisons. Among 3 different configurations of tension band wiring techniques for adult olecranon fractures, the olecranon screw group or Kirschner-wire group had a relatively higher satisfactory rate than the rush pin group. The authors concluded that for olecranon fractures with a large fragment, olecranon screw or Kirschner wire can be chosen. For olecranon fractures with a small fragment, Kirschner wire should be utilized. ${ }^{13}$

This study is directed towards the clinical evaluation of results of tension band technique for olecranon fractures using cancellous screws and $\mathrm{K}$ wires and to compare the results.

\section{METHODS}

The present study consists of 20 cases of fracture olecranon treated by Tension band wiring technique, patients were randomly divided into two groups, one study group of 10 patients were operated with TBW using Kirshner wires and the other study group of 10 patients were operated with TBW using $6.5 \mathrm{~mm}$ cancellous screw and procedures were done at Chigateri General Hospital and Bapuji Hospital, Davangere, India, between August 2012 to November 2013.

\section{Inclusion criteria}

- Adults

- Transverse or oblique fractures

- No comminution or minimally comminuted fractures

\section{Exclusion criteria}

- Extremes of age

- Comminuted fractures

- Grossly contaminated and infected open fractures

- Associated distal humeral fractures

- Fractures with neurovascular deficits

\section{Surgical procedure}

\section{TBW with $K$-Wire (Figure 1)}

The operation was performed under general anaesthesia or brachial plexus block. After mid arm tourniquet was applied with patient in supine or lateral position site of the surgery was thoroughly painted with iodine and spirit and draped. Exposure of the olecranon was done by Campbell's approach. Accurate anatomical reduction was achieved and held with either reduction clamp or long towel clip.

Two K-wires is introduced parallel from the tip of the olecranon i.e., the proximal fragment across the fracture 
site to the distal fragment piercing the anterior cortex. Periosteum was stripped from the shaft of ulna distal to fracture site and a transverse hole was drilled approximately 3 to $5 \mathrm{cms}$ distal to fracture site. A No 18 stainless steel malleable wire was passed through this transverse hole and crossed over the posterior surface of olecranon in a figure-of-eight manner and then passed around the protruding Kirschner wires and tightened using AO tensioner and then secured with a twist. Bend the proximal ends of the Krishner wires 1800 and tap the cut ends back into the proximal fragment. Accuracy of reduction was checked and stability was tested by moving the joint. Wound closed in layers and sterile dressing and compression bandage given.

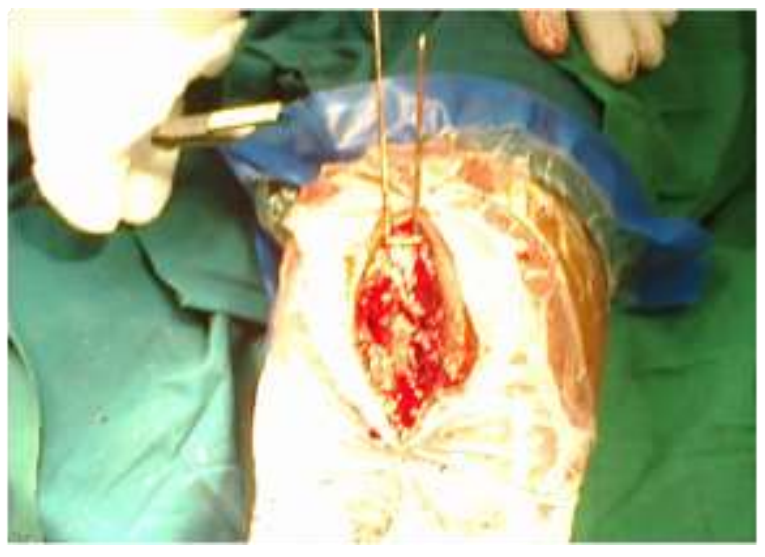

Figure 1: Tightened SS wire with $K$ wire across the fracture.

\section{TBW with Cancellous screw (Figure 2)}

With similar anaesthesia, position, tourniquet application and approach, accurate anatomical reduction was achieved and held with reduction clamp or towel clip. 6.5 $\mathrm{mm}$ AO cancellous screw is introduced parallel from the tip of the olecranon across the fracture site to the distal fragment. A no 18 stainless steel malleable wire is applied in a figure of eight manner and then passed around the neck of the screw and tightened using AO tensioner and then secured with a twist. Accuracy of reduction was checked and stability was tested by moving the joint. Wound was closed in layers and sterile dressing and compression bandage given.

Postoperatively all the patients were treated with antibiotics for five days and analgesics. Affected limb was elevated and patient was asked to perform finger movements on day 1. Elbow movements were advised from $3^{\text {rd }}$ postoperative day.

Patients were followed up at 6 weeks and 12 weeks and thereafter every 3 months. The result was assessed 3 months after the procedure. At follow up a detailed clinical examination was done and patient was assessed subjectively for the symptoms like pain, swelling, restriction of joint motion. On clinical examination, swelling of the joint, tenderness, movements of the elbow joint, prominence of head of cancellous screw and prominence of $\mathrm{K}$ wire, nutrition and power of the muscles acting on the joint were noted.

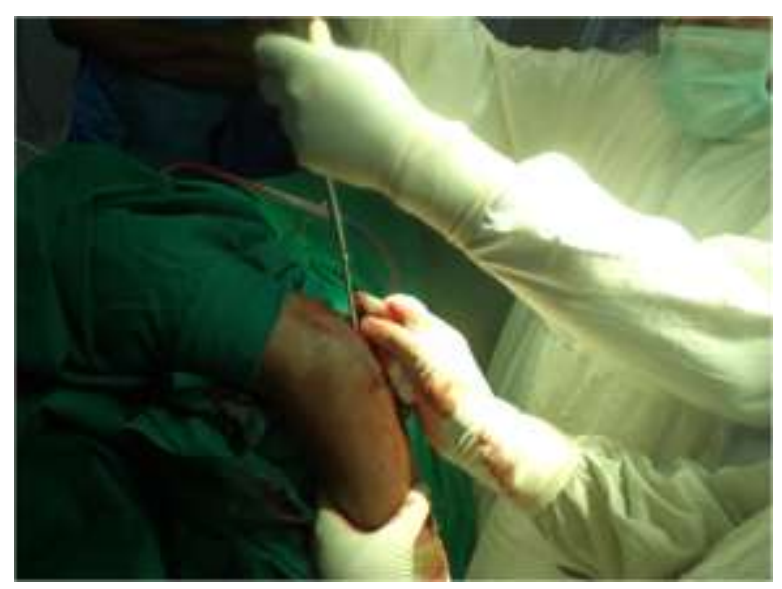

Figure 2: 6.5 Cancellous screw insertion across the fracture.

Patients were instructed to carry out physiotherapy in the form of, active flexion-extension and pronationsupination without loading. Check x-ray were taken at each follow-up. In all patients duration after which they returned to job was noted.

The results were evaluated in our study with Mayo Elbow Performance score (MEPS) for functional outcome and Standard radiographs for radiological outcome (Table 1). ${ }^{14}$

Table 1: Interpreting the Mayo elbow performance score.

\begin{tabular}{|llll|}
\hline $\begin{array}{l}\text { Greater } \\
\text { than } 90\end{array}$ & $\begin{array}{l}\text { Score } 75 \text { to } \\
89\end{array}$ & $\begin{array}{l}\text { Score } 60 \text { to } \\
74\end{array}$ & $\begin{array}{l}\text { Score } \\
\text { below } 60\end{array}$ \\
\hline Excellent & Good & Fair & Poor \\
\hline
\end{tabular}

\section{RESULTS}

Study consists of 20 patients with olecranon fractures treated at Chigateri General Hospital and Bapuji Hospital attached to J.J.M. Medical College, Davangere, India, between August 2012 to November 2013. Patients were randomly divided into two groups of equal number (10 in each group) where patient with odd number were treated using tension band wiring ( $\mathrm{TBW}$ ) with ' $\mathrm{K}$ ' wire and those with even number are treated by tension band wiring with cancellous screw. All patients were followed up periodically during the period 2012-2013. The following are the observations made and available data are analysed as follows.

The mean age of patients treated by cancellous screw with tension band wiring was 39.2 \pm 11 years (range 23-58 years) while those treated by tension band wiring with $\mathrm{K}$ wire patients mean age was 37.6 \pm 16.1 years (range $21-50$ years). 
Both groups had equal number of male and female patients i.e. seven males (70\%) and three females (30\%).

In group treated by cancellous screw with tension band wiring eight patients $(80 \%)$ had right olecranon fracture, 2 patients $(20 \%)$ had left side olecranon fracture. In the group treated by tension band wiring with $\mathrm{K}$ wire six patients $(60 \%)$ had right olecranon fracture and four patients $(40 \%)$ had left side olecranon fracture.

The most common mechanism of injury in both groups was fall on the elbow, eight patients $(80 \%)$ on TBW with cancellous screw group and seven patients (70\%) in TBW with K-wire group. Two patients were injured in road traffic accidents (20\%) in each group and one patient $(10 \%)$ had history of assault in TBW with K-wire group.

In the TBW with cancellous screw group seven patients $(70 \%)$ had transverse fracture and three patients $(30 \%)$ had oblique fracture. In TBW with K-wire group six patients $(60 \%)$ had transverse fractures and three $(30 \%)$ had oblique fracture and one patient $(10 \%)$ had avulsion fractures (Table 2). In tension band wiring with $\mathrm{K}$-wire group one patient had fracture radial head $(10 \%)$ and nine patients $(90 \%)$ had isolated olecranon fracture. In the TBW with cancellous screw group only one patient $(10 \%)$ had fracture shaft of ulna and the remaining nine patients $(90 \%)$ had isolated olecranon fracture.

Table 2: Type of fractures (Colton's classification). ${ }^{15}$

\begin{tabular}{|lll|l|}
\hline $\begin{array}{l}\text { Type of } \\
\text { fractures }\end{array}$ & $\begin{array}{l}\text { TBW with K } \\
\text { wire } \\
\mathbf{n}(\%)\end{array}$ & $\begin{array}{l}\text { TBW } \\
\text { with } \\
\text { cancellou } \\
\text { s screw } \\
\mathbf{n}(\%)\end{array}$ & $\begin{array}{l}\text { Total } \\
\mathbf{n}(\%)\end{array}$ \\
\hline Transverse & $6(60)$ & $7(70)$ & $13(65)$ \\
\hline Oblique & $3(30)$ & $3(30)$ & $6(30)$ \\
\hline Avulsion & $1(10)$ & - & $1(5)$ \\
\hline Total & 10 & 10 & $20(100)$ \\
\hline
\end{tabular}

Chi-square $=0.39, \mathrm{p}=0.53, \mathrm{NS}$

Patients were operated with an average period of 3 to 4 days after the injury and the mean postoperative days for tension band wiring with $\mathrm{K}$-wire was $6.9 \pm 1.4$ days and for tension band wiring with cancellous screw group mean postoperative days were $6.4 \pm 1.2$ days. Check $\mathrm{x}$ rays (both antero-posterior and lateral views) obtained after immediate postoperative, 6 weeks and 12 weeks and then follow up done monthly till the end of follow up period. In the TBW with $\mathrm{K}$-wire group three patients (30\%) came for follow up for 3 months, one patient (10\%) followed up for 5 months, and six patients $(60 \%)$ were followed up for 6 months and in TBW with cancellous screw four patients $(40 \%)$ were followed up 4 months, three patients $(30 \%)$ had 5 months and another three patients $(30 \%)$ were followed up for six months. In all the patients the duration after which they returned to their job were noted.
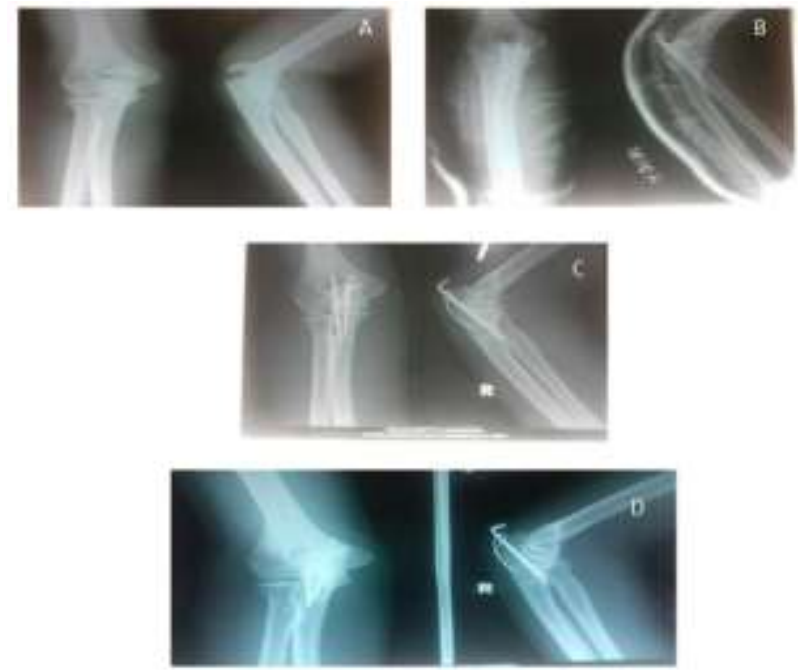

Figure 4: A- Pre op. X-ray; B- Immediate Post op. Xray; C- Check X-ray after 6 weeks; D- Check X-ray after 12 weeks.
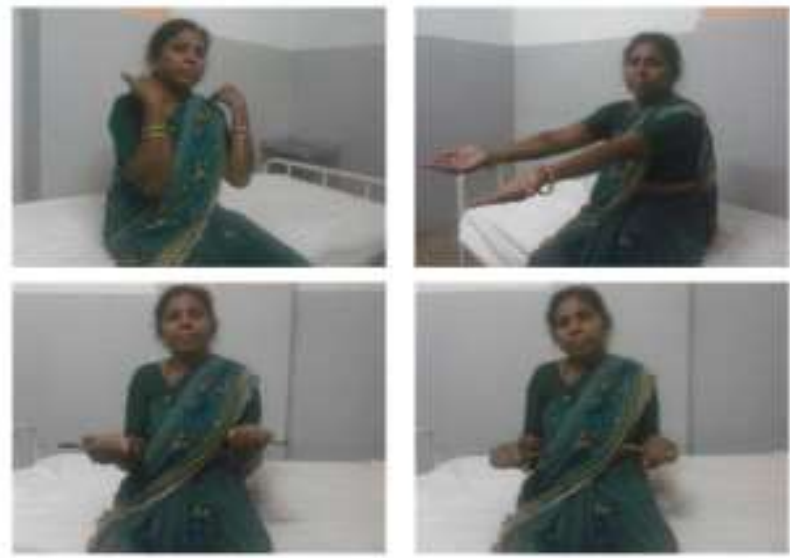

Figure 5: Complete range of motion at 3 months follow up operated with $\mathrm{K}$ wire and TBW.
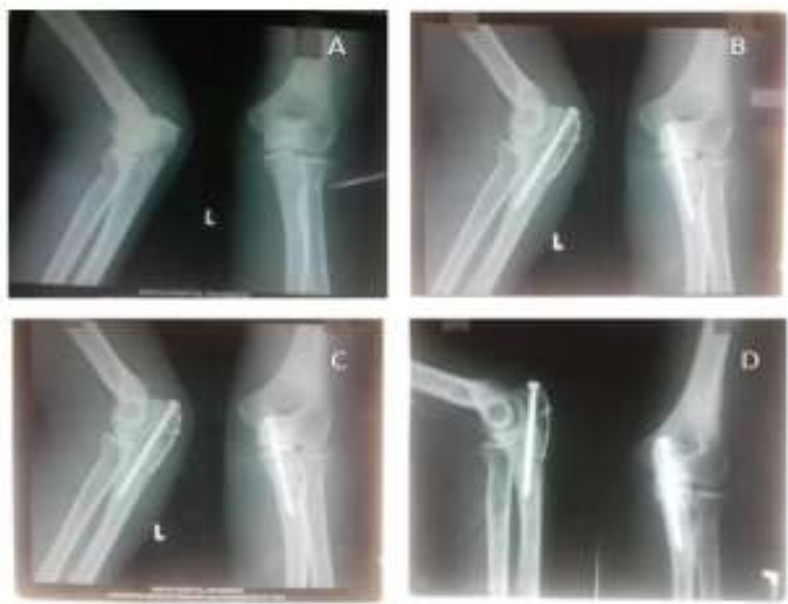

Figure 6: A- Pre op. X-ray; B- Immediate Post op. Xray; C- Check X-ray after 6 weeks; D- Check X-ray after 12 weeks. 
In the TBW with K-wire group four patients (40\%) had fracture union in 12 weeks, in four patients (40\%) union achieved in 14 weeks and remaining two patients $(20 \%)$ took 16 weeks for union of the fracture. However in the group TBW with cancellous screw group, seven patients (70\%) showed fracture union in 12 weeks remaining three patients $(30 \%)$ had fracture union in 14 weeks.

At the end of the follow up patients were assessed for pain. In the TBW with cancellous screw group, eight patient $(80 \%)$ had no pain and two patients $(20 \%)$ had mild pain and in the TBW with $\mathrm{K}$-wire group five patients $(50 \%)$ were pain free and five patients $(50 \%)$ had mild aching pain.

In the present study in the group TBW with cancellous screw nine patients $(90 \%)$ showed good arc of motion greater than $100^{\circ}$ and one patient $(10 \%)$ was having arc of motion between $50^{\circ}$ and $100^{\circ}$. In the group TBW with $\mathrm{K}$-wire seven patients (70) were having arc of motion greater than $100^{\circ}$ and three patients $(30 \%)$ showed arc of motion between $50^{\circ}$ and $100^{\circ}$.
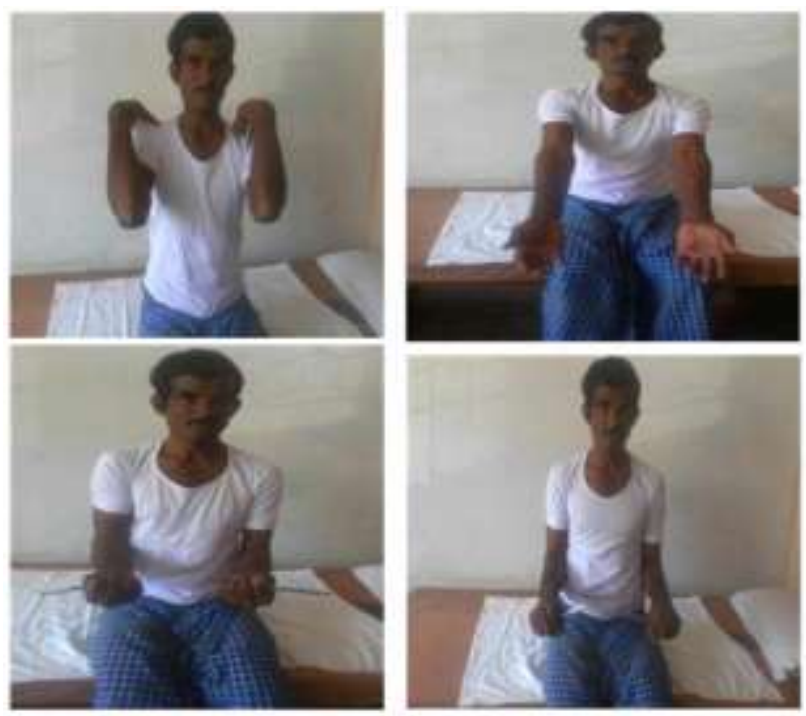

Figure 7: Complete range of motion at 3 months follow up operated with cancellous screw and TBW.

In the TBW with K-wire group eight patients (80\%) having stable elbow and two patients $(20 \%)$ had moderate instability and in the TBW with cancellous screw group all patients $(100 \%)$ were having stable elbow.

In this study $\mathrm{TBW}$ with cancellous screw group nine $(90 \%)$ were able to perform the functions as per the Mayo elbow performance chart and only one patient $(10 \%)$ was not able to perform one function. In the TBW with $\mathrm{K}$ wire group eight patients $(80 \%)$ were able to perform the functions and two patients $(20 \%)$ were not able to perform one function.
In the TBW with K-wire group 3 patients (30\%) showed prominence of the hardware requiring second surgery for removal of prominent hard ware and one patient $(10 \%)$ had superficial infection which was treated with broad spectrum antibiotic.

Table 3: Observation.

\begin{tabular}{|llll|}
\hline Results & $\begin{array}{l}\text { TB W } \\
\text { with K } \\
\text { wire } \\
\text { n }(\%)\end{array}$ & $\begin{array}{l}\text { TB W with } \\
\text { cancellous } \\
\text { screw } \\
\text { n (\%) }\end{array}$ & $\begin{array}{l}\text { Total } \\
\text { n }(\%)\end{array}$ \\
\hline $\begin{array}{l}\text { Excellent } \\
(\text { score >90) }\end{array}$ & $5(50)$ & $8(80)$ & $13(65)$ \\
\hline $\begin{array}{l}\text { Good } \\
\text { score 75-89) }\end{array}$ & $3(30)$ & $1(10)$ & $4(20)$ \\
\hline $\begin{array}{l}\text { Fair } \\
(\text { score 60-74) }\end{array}$ & $2(20)$ & $1(10)$ & $3(15)$ \\
\hline Poor & 0 & 0 & 0 \\
\hline Total & 10 & 10 & $\begin{array}{l}20 \\
(100)\end{array}$ \\
\hline
\end{tabular}

$\mathrm{X}^{2}=2.03, \mathrm{p}=0.36, \mathrm{NS}$

In the TBW with cancellous screw group only one patient (10\%) had prominence of hardware requiring second operation for removal of prominent hardware.

In the TBW with cancellous screw eight patients $(80 \%)$ showed excellent result, one patient $(10 \%)$ had good and one patient $(10 \%)$ had fair result. In the TBW with K-wire group five patients $(50 \%)$ had excellent results and three patients $(30 \% \mathrm{P})$ had good and two patients (20\%) had fair result (Table 3). In the TBW with cancellous screw group only one patient (10\%) had prominence of hardware requiring second operation for removal of prominent hardware. However poor results were not seen in both the groups.

Table 4: According to Mayo elbow performance score.

\begin{tabular}{|lllll|}
\hline Method & $\begin{array}{l}\text { Excellent } \\
\text { n(\%) }\end{array}$ & $\begin{array}{l}\text { Good } \\
\text { n(\%) }\end{array}$ & $\begin{array}{l}\text { Fair } \\
\text { n }(\%)\end{array}$ & $\begin{array}{l}\text { Poor } \\
\text { n }(\%)\end{array}$ \\
\hline $\begin{array}{l}\text { TBW with } \\
\text { K-wire }\end{array}$ & $5(50)$ & $3(30)$ & $2(20)$ & - \\
\hline $\begin{array}{l}\text { TBWwith } \\
\text { cancellous } \\
\text { screw }\end{array}$ & $8(80)$ & $1(10)$ & $1(10)$ & - \\
\hline
\end{tabular}

\section{DISCUSSION}

The treatment of fractures of the olecranon has seen the gamut from early range of motion of the elbow without regard for the fracture to precise and open anatomic reduction of the fracture site.

Before the era of aseptic surgery and the discovery of roentgenography, olecranon fractures were treated by splinting the elbow in full extension for 4 to 6 weeks 3 . This usually resulted in a stiff elbow with loss of flexion. Later the practitioners slowly began to use the position of 
mid-flexion but, this frequently led to nonunion because of wide separation of fracture fragments, resulting in decreased power of the triceps mechanism. ${ }^{16}$

The dilemma for nonunion and stiffness led Lister to choose the fracture of olecranon to be the first fracture treated by open reduction and internal fixation using his method of asepsis with a wire loop. ${ }^{3}$ Modifications of this technique, which was the forerunner of the tension band technique advocated by the $\mathrm{AO}$ group are now in use.

The main aim of the treatment of olecranon fractures is not only achieving union but to preserve the optimum function of the adjacent soft tissues and joints. In the management of intra-articular fractures like fracture of olecranon, a perfect anatomical reduction of the fragments to obtain articular congruity and rigid fixation of the fragments is of utmost importance, if early movements are to be instituted to prevent complications like traumatic arthritis and joint stiffness.

The dynamic compression is one where the fragments are not only compressed by pre-stress of the implant but also are subjected to additional compression which results from harnessing forces generated at the level of the fracture when skeleton comes under normal physiological load.

It was Pauwel who first borrowed from industrial mechanics of the principle of tension band fixation and demonstrated its application in internal fixation of bone. Every eccentrically loaded bone is subjected to bending stresses. This results in typical distribution of stresses with tension on the convex and compression on the concave side of the bone. This is also why when such a bone fractures it displaces with a gap on the tension side. In order to restore the load bearing capacity of an eccentrically loaded fractured bone, the tensile forces have to be absorbed by a tension band wire and the bone itself has to be able to withstand axial compression. The pre stressing of the device in tension results in inter fragmental compression. The loading results in a dynamic increase of this axial inter fragmental compression.

Hence, summarizing the whole principle of tension band in a sentence, it is well said that "The implant absorbs the tension and the bone the compression".

Patrica Villaneva treated 37 cases of olecranon fractures with Tension band wiring and stated Tension band wiring provided excellent results in a high percentage of olecranon fractures. ${ }^{17}$ In Byron E Chalidis et al, reviewed 62 patients of isolated olecranon fractures and came to a conclusion that tension band wiring technique is the "Gold standard" for the treatment of olecranon fractures and leads to good elbow function and minimal loss of physicalcapacity. ${ }^{18}$

Tension band wiring with $\mathrm{K}$-wire is the most common method for fixation of olecranon fractures and it works on the principle of converting tensile forces to compressive forces at fracture site, it usually gives good result but cancellous screws with tension band wiring combination provides the strength of fixation that is by converting the tensile force to a compressive force at the fracture site with additional resistance to the displacement due to the lag screw compression.

In our study 20 cases of olecranon fractures were treated out of which 10 cases were fixed with tension band wire with $\mathrm{K}$-wire and 10 cases are fixed with cancellous screw with tension band wire, results of the two groups are studied and our experience with second group has given favorable results.

The findings end results and various other data will be analyzed and compared in the following discussion.

The average age incidence in the present study for TBW with K-wire is 37.6 years and TBW with cancellous screw is 39.2 years

In the present study of fracture olecranon greater incidence is seen in males. We found that the involvement of right side fracture in 6 patients $(60 \%)$ and left in 4 patients $(40 \%)$ in the TBW with $\mathrm{K}$-wire group and in TBW with cancellous screw group, in 8 patients (80\%) fracture seen on right side and 2 patients $(20 \%)$ had fracture on left side. Most common mode of injury in both groups was fall on the elbow i.e. $8(80 \%)$ in TBW with cancellous group and $7(70 \%)$ in TBW with K-wire group, 2 patients $(20 \%)$ were injured in RTA and only one patient $(10 \%)$ patient sustained fracture due to assault in TBW with $\mathrm{K}$-wire group.

In TBW with K-wire group $6(60 \%)$ patients had transverse fracture and $3(30 \%)$ patients had oblique fracture and $1(10 \%)$ patient was having avulsion fracture and $1(10 \%)$ patient had associated injury of radial head fracture. In the TBW with cancellous screw group, 7 $(70 \%)$ patients had transverse fracture and $3(30 \%)$ patients had oblique fracture and one $(10 \%)$ patient had associated fracture shaft of ulna.

In the present study $3(30 \%)$ patients showed hardware prominence seen in TBW with $\mathrm{K}$-wire group requiring second operation for removal of implant and only one (10\%) patient showed hardware prominence in TBW with cancellous screw group.

Results were evaluated according to the Mayo elbow performance score. According to Mayo elbow performance score, $8(80 \%)$ patients in our study had excellent results in TBW with cancellous screw group and 1 (10\%) patient each had good and fair result respectively and no patient had poor result. ${ }^{14}$ In the TBW with K-wire group $5(50 \%)$ patient had excellent results, $3(30 \%)$ patients had good results and $2(20 \%)$ patients had fair results and no patients had poor results (Table 3). 


\section{CONCLUSION}

From the present study it is concluded that using cancellous screw with tension band wiring for displaced transverse and oblique olecranon fractures gives better clinical results and has much less re-operation rate for removal of hardware when compared to tension band wiring with $\mathrm{K}$-wire fixation avoiding cost, work time loss and possible complications from hard ware removal.

Funding: No funding sources

Conflict of interest: None declared

Ethical approval: Not required

\section{REFERENCES}

1. Parker MJ, Richmond PW, Andrew TA, Bewes PC. A review of displaced olecranon fractures treated conservatively. J R Coll Surg Edinb. 1990;35(6):392-4.

2. Ring D. Elbow fractures and dislocations. Rockwood and green fractures in adults. Vol I 7th, In: Bucholz RW, Heckman JD eds. Lippincott Williams \& Wilkins. 2010;936-42.

3. Howard JL, Urist MR. Fracture dislocation of the radious and the ulna at the elbow joint. Clin Orthop. 1958;12:276-84.

4. Eliot E. Fracture of the olecranon. Surg Clin North Am. 1934;(14):487-92.

5. Daland EM. Fractures of the olecranon. J Bone Joint Surg. 1933;15:601-7.

6. Holdsworth BJ, Mossad MM. Elbow function following tension band fixation of displaced fractures of the olecranon. Injury. 1984;16:182-7.

7. Weber BG, Vasey H. Osteosynthesis bei olecranon frakur. Rev Accid Trav Mal Prol. 1963;56:90.

8. Cooper, Jerald L, D'Ambrosia Robert D. Fracture and fracture dislocation about the elbow. Operative Orthopaedics. Vol I, $2^{\text {nd }}$ Edn. Chapman Michoel WJB. Philadelphia: Lippincott Company; 1993:479482.
9. Willams JR. Coronoid, radial head, olecranon fractures and elbow dislocations. Vol 3. Oxford Text book of Orthopaedics and Trauma. OUP UK; 2002:1969-1972.

10. Crenshaw, Andrew H. Fractures of shoulder, arm and forearm. Campbell's operative orthopadeics. Vol 3, $11^{\text {th }}$ Edn, Terry CS, Beaty JH. Mosby; 2008:3411-3417.

11. MacAusland WR. The treatment of the olecranon by longitudinal screw or nail fixation. Ann Surg. 1942;116:293-6.

12. Murphy DF, Greene WB, Gilbert JA, Dameron TB. Displaced olecranon fractures in adults. Biomechanical analysis of fixation methods. Clin Orthop. 1987;224:210-4.

13. Fan GF, Wu CC, Shin CH. Olecranon fractures treated with tension band wiring techniquescomparisons among three different configurations. Changgeng Yi Xue Za Zhi. 199;16(4):231-8.

14. Morrey BF, An KN. Functional evaluation of the elbow. In: Morrey BF, editor. The elbow and its disorders. $3^{\text {rd }}$ edition Philadelphia: WB Saunders; 2000:82.

15. Colton CL. Fractures of the olecranon in adults: classification and management. Injury. 1973;5:1219.

16. Perkins G. Fractures of the olecranaon. Br Med J Clin Res. 1936;2:668-9.

17. Patrica Villaneva, et al: Tension band wiring for olecranon fractures, Analysis of risk factors for failure. J Shoulder and Elbow Surgery. 2006;15(3):351-6.

18. Chaldis BE, Sachinis NC, Samoladas EP. Is tension band wiring technique the "gold standard" for the treatment of olecranon fractures. J Orthopaed Surg Res. 2008;3:9.

Cite this article as: Krishnaprasad HS, Shivanna. Comparative study of management of olecranon fractures using tension band wiring technique with cancellous screw and $\mathrm{K}$ wires. Int $\mathrm{J}$ Res Orthop 2016;2:109-15. 